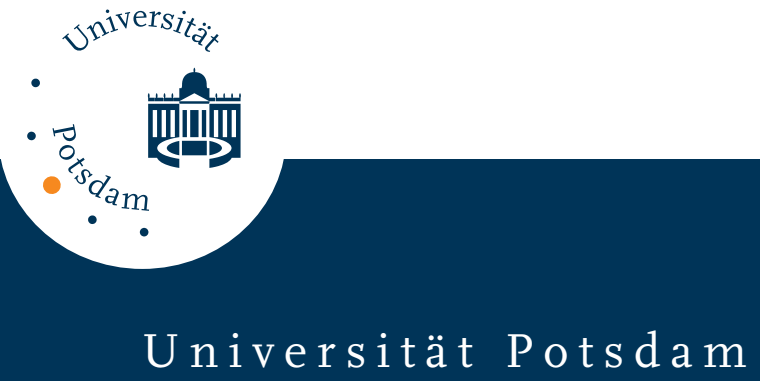

Reinhold Kliegl, Richard K. Olson, Brian J. Davidson

\title{
On problems of unconfounding perceptual and language processes
}

first published in:

Eye movements in reading : perceptual and language processes / ed. by Keith Rayner. - New York [u.a.] : Acad. Press, 1983. - (Perspectives in neurolinguistics, neuropsychology, and psycholinguistics), S. 333-343

Postprint published at the Institutional Repository of the Potsdam University: In: Postprints der Universität Potsdam

Humanwissenschaftliche Reihe ; 140

http://opus.kobv.de/ubp/volltexte/2009/3989/

http://nbn-resolving.de/urn:nbn:de:kobv:517-opus-39890

Postprints der Universität Potsdam

Humanwissenschaftliche Reihe ; 140 


\section{9 \\ On Problems of \\ Unconfounding Perceptual and Language Processes}

\section{Introduction}

Over the past decade we have witnessed a major revival of research on eye fixations in reading. In contrast to earlier work (e.g., Tinker, 1958), which was concerned with questions of average fixation duration and frequency, most recent studies have focused on factors influencing the durations and locations of individual fixations, and on the amount of information available during a single fixation (cf. McConkie, 1979; O'Regan, 1979; Rayner, 1979b). Although the influence of psycholinguistic variables has been demonstrated in some of these studies, more attention has been directed toward perceptual factors associated with acuity limitations in eye guidance. One could conclude from this research that acuity limitations play a dominant role in length of saccades and placement of fixations on words.

Just and Carpenter (1980) evaluated eye fixation data from a very different perspective. They examined the influence of psycholinguistic variables on eye fixations, and perceptual variables were not considered. Word length, which would play a dominant role from a perceptual point of view, was coded by number of syllables in their psycholinguistic model. Kliegl, Olson, and Davidson (1982) demonstrated that some of Just and Carpenter's (1980) psycholinguistically relevant variables, such as number of syllables, number of letters, and word frequency, are highly correlated with each other in natural text. Hierarchical regression analyses of our data showed that the amount of variance in fixation time explained independently by these variables is small compared to the amount of variance they share. When word length in letters rather than syllables was entered in the analyses, the fit of the model improved. Moreover, the independent contribution 
of syllable length was nonexistent, and the independent contribution of word frequency decreased from 12 to $3 \%$. If one were to interpret word length in number of letters as a perceptual indicator and word length in number of syllables as a psycholinguistic process indicator, this result would lend support to the relevance of perceptual processing.

The use of eye fixation data for theory development in reading will require a better separation of perceptual and psycholinguistic influences. In natural text, variables that may be theoretically related to psycholinguistic and perceptual processes, like word length and word frequency, are highly correlated or-as is the case for word length - may be claimed for both types of processes. Consequently, multiple regression analyses employing these indicators will not provide much insight into the relative importance of the underlying processes.

One way to avoid correlations in natural text is via traditional experimental designs. The advantage of the experimental approach lies in the strict orthogonality of the factors. The cost of the clean design is the artificiality of the experimental material. Further, since experimental conditions are usually conceptualized as fixed factors, ANOVA designs cannot provide process parameters; they only allow us to ascertain that a certain factor is significantly related to the dependent measure. Nevertheless, it seems important to assess the presence of these effects in natural text. Therefore, we have adopted an alternative dataanalytic approach that introduces orthogonality post hoc by selective sampling in the data base. This approach isolated different perceptual and psycholinguistic influences by holding one variable constant in the sample and noting fixation time variance with the other.

The data used in the following analysis included various subsets of the data used for the global regression analysis reported by Kliegl (1981b). In this experiment six skilled adult readers carefully read the beginning pages (1260 words) of Camus's novel The Plague (1947/1948). Eye fixations were monitored with an Applied Sciences Eye View Monitor Model 1996. This is a video based system with a sampling rate of $60 \mathrm{~Hz}$ and an accuracy of about $1 / 2^{\circ}$ of visual angle. Character position accuracy was within one character to the right or left of the fixated character at least $90 \%$ of the time (see Davidson, 1981; Kliegl, 1981a; Kliegl \& Olson, 1981 for technical details).

Two sets of analyses were performed to test for the influence of perceptual and psycholinguistic processes on the number, duration, and placement of fixations on a word. The first set separated the effects of syllable- and letter-length of words and their frequency. To this end, words were selected to conform to an orthogonal design with syllable length (SL) and letter length (LL) as independent factors. The second set replicated previous experimental findings on fixation durations and positions of fixations. The analyses were carried out separately for words that received one fixation and words that received two fixations. The two-fixation cases were also analyzed for differences between regressive sequences and progressive sequences of fixations within words. 


\section{Word Length and Word Frequency}

One of the issues central to the development of an eye fixation based process theory of reading is how to disentangle the influence of perceptual and psycholinguistic factors. The regression analyses performed by Kliegl et al., (1982) suggested a further examination of the relation between word length and word frequency, as these were the parameters contributing most to the fit of the model. Variance contributed by word frequency can be taken to reflect psycholinguistic processing demands. The length of the word might be taken to reflect psycholinguistic factors from the point of view of syllables (Just \& Carpenter, 1980; Pynte, 1974), and eye guidance, peripheral acuity, or letter processing from the perspective of number of letters. In the hierarchical regression analyses discussed earlier, number of syllables did not contribute to fixation time independently of number of letters, and the independent contribution of word frequency was small. However, the type of data entered in those models might have buried independent contributions of these two parameters. The data were gaze durations, that is, the total time spent on a word (cf. Just \& Carpenter, 1980). Gaze duration reflects the number of fixations on a word as well as fixation duration per se. To illustrate, suppose that a given word is fixated only $50 \%$ of the time, but when it is fixated the durations are $250 \mathrm{msec}$ (approximately the average fixation time). Gaze duration for that word would be $125 \mathrm{msec}$. Similarly, consider a word that receives two fixations half of the time, and one fixation the other half. If each fixation again lasts $250 \mathrm{msec}$, the gaze duration would be $375 \mathrm{msec}$, even though for a given subject the total fixation time would be either 250 or $500 \mathrm{msec}$. Thus, gaze duration cannot disentangle the number of fixations on a word from fixation durations per se. In fact, a large amount of the variance in our earlier analyses was due to words that were not fixated or that were fixated more than once. Skipped words tended to be short and of high frequency, and multiple fixation words tended to be long and of low frequency.

To unconfound the number of fixations from fixation duration, we focused on words that received only a single fixation. Words were selected from the text to conform to a post-hoc orthogonalized design. Only words 5-11 letters long were selected. This restriction ensured that there were enough words in the central cells of the letters $(5,11)$ by syllables $(1,4)$ matrix. Also, an upper cutoff of 500 msec was used to remove some outliers.

The average fixation durations for the various letter-syllable combinations, standard deviations, and number of observations are shown in Table 19.1. The rightmost column will be discussed later. There was no significant influence of either letter length or syllable length on the fixation durations. Thus, when a word receives a single fixation, the fixation duration is essentially unrelated to word length. Notice that all letters of these words would be well within the span of perceptual acuity. One might expect double fixations on longer words, because of acuity limitations. From a psycholinguistic perspective, however, this result 
TABLE 19.1

Descriptive Statistics for One-Fixation Case (OFC) ${ }^{a}$

\begin{tabular}{|c|c|c|c|c|c|c|}
\hline \multirow{2}{*}{$\begin{array}{l}\text { Number of } \\
\text { letters }\end{array}$} & & \multicolumn{4}{|c|}{ Number of syilables } & \multirow{2}{*}{$\begin{array}{l}\text { Letter position } \\
\text { of fixation }\end{array}$} \\
\hline & & 1 & 2 & 3 & 4 & \\
\hline \multirow{3}{*}{5} & FT & 250 & 235 & - & 一 & 2.8 \\
\hline & (s) & (75) & $(80)$ & - & - & $(1.6)$ \\
\hline & $N$ & 318 & 91 & - & - & 409 \\
\hline \multirow{3}{*}{6} & FT & 245 & 257 & - & - & 3.0 \\
\hline & (s) & $(73)$ & $(85)$ & - & - & $(1.8)$ \\
\hline & $N$ & 79 & 226 & - & - & 305 \\
\hline \multirow{3}{*}{7} & FT & - & 255 & 235 & - & 3.3 \\
\hline & $(s)$ & - & $(77)$ & (87) & - & $(2.0)$ \\
\hline & $N$ & - & 186 & 47 & - & 233 \\
\hline \multirow{3}{*}{8} & $\mathrm{FT}$ & - & 260 & 253 & - & 3.6 \\
\hline & $(\mathrm{s})$ & - & $(95)$ & $(78)$ & - & $(2.1)$ \\
\hline & $N$ & - & 56 & 91 & - & 147 \\
\hline \multirow{3}{*}{9} & $\mathrm{FT}$ & - & 287 & 248 & - & 4.4 \\
\hline & (s) & - & (93) & (85) & - & $(2.3)$ \\
\hline & $N$ & - & 30 & 49 & - & 79 \\
\hline \multirow{3}{*}{10} & $\mathrm{FT}$ & - & - & 235 & 247 & 4.2 \\
\hline & (s) & - & - & (97) & (90) & $(2.6)$ \\
\hline & $N$ & - & - & 18 & 20 & 38 \\
\hline \multirow{3}{*}{ II } & $\mathrm{FT}$ & - & - & 257 & 223 & 4.2 \\
\hline & (s) & - & - & (118) & (92) & $(2.9)$ \\
\hline & $N$ & - &. & 12 & 9 & 21 \\
\hline
\end{tabular}

${ }^{a} \mathrm{FT}$ is fixation time (msec); $\mathrm{s}$ is standard deviation (msec); $N$ is number of data points.

calls into question any assumption of serial processing of subword units based on number of letters or syllables (Gough, 1972; Just \& Carpenter, 1980) and favors a whole-word processing hypothesis (Cattell, 1885; McClelland \& Rumelhart, 1981).

Given that word length showed no relation to fixation duration, we then explored the influence of word frequency. The critical correlation, based on the same data set, was significant but rather low at .11.

The conclusion from the above analyses is that a substantial portion of the variance in gaze duration is attributable to the number of fixations on a word, rather than to fixation duration per se. In view of these results, the assumption that gaze duration reflects processing time seems unwarranted. However, it is possible that some words receive two fixations because they are more difficult to process. The following analyses, therefore, attempted to separate perceptual and psycholinguistic influences on fixation frequency. 


\section{Preferred and Convenient Viewing Position}

Strong support for the relevance of eye guidance for process theories of reading comes from the finding that fixations tend to be located slightly to the left of the middle of the word (Rayner, 1979a). For this to occur, some peripheral preprocessing must take place. Once the eye obtains this preferred viewing position, the whole word can be better apprehended within a single fixation. The right column in Table 19.1 gives the average letter position for words varying from 5 to 11 letters in length. Note that with increasing word length, the average fixation position moves further toward the right, confirming Rayner's results.

Recently, O'Regan (1980, 1981) demonstrated that under conditions of nonoptimal positioning of the eye, that is, close to the beginning or the end of the word, distributed processing is likely to occur. That is, a second fixation tends to be placed at the other end of the word. In this case, the normally preferred position is not taken. These findings can be tested in a post-hoc fashion with the present data. The question is whether fixation positions in the word differ significantly given one or two fixations.

We have examined all double- and single-fixation words five to nine letters long in our sample of text. Pooling across subjects and the selection criteria made statistical tests problematic. The same word could possibly appear in different categories. Other words might show up only once, or the same word might show up a different number of times in different categories. Analyses were made under the assumption that the words in the one-fixation case, in the progressive sequence, and in the regressive sequence form independent groups. The average fixation duration for the one-fixation case (OFC) was $253 \mathrm{msec}$. For the twofixation case (TFC), the first fixation in a progressive sequence (PS) was 248 msec. The first fixation in a regressive sequence (RS) averaged $214 \mathrm{msec}$. The respective second fixations lasted $223 \mathrm{msec}$ and $237 \mathrm{msec}$ on the average. The left half of Table 19.2 displays these values and the corresponding number of observations and standard deviations. The OFC resulted in significantly longer fixation durations; fixation durations for PS and RS were not statistically different. Note that the values for PS and RS were based on the averages of two fixations occuring in these patterns. These results confirm, in continuous text, O'Regan's finding that single fixations on a word are typically longer than each of two fixations in the two-fixation case. Notice, however, that a gaze measure, such as the sum of the single fixation durations, would yield almost twice as much processing time under TFC.

The right half of Table 19.2 also shows the letter positions at which the various fixations occurred. The space in front of a word was interpreted as the 0-letter position. The following order describes the positions of the fixations across the different conditions: first fixation of PS, second fixation of RS, fixation of OFC, 
TABLE 19.2

Mean (Standard Deviation) of Fixation Duration and Letter Position for One and Two Fixation Case

\begin{tabular}{|c|c|c|c|c|}
\hline \multirow[b]{2}{*}{ Case } & \multicolumn{2}{|c|}{ Duration } & \multicolumn{2}{|c|}{ Position } \\
\hline & First fixation & Last fixation & First fixation & Lost fixation \\
\hline $\begin{array}{l}\text { One fixation case } \\
(N=1116)\end{array}$ & $\begin{array}{l}253 \\
(82)\end{array}$ & - & $\begin{array}{l}3.5 \\
(1.7)\end{array}$ & - \\
\hline $\begin{array}{l}\text { Two fixation cases } \\
\text { Progressive sequence } \\
(N=166)\end{array}$ & $\begin{array}{l}248 \\
(93)\end{array}$ & $\begin{array}{l}223 \\
(87)\end{array}$ & $\begin{array}{c}1.3 \\
(1.3)\end{array}$ & $\begin{array}{c}5.5 \\
(2.0)\end{array}$ \\
\hline $\begin{array}{l}\text { Regressive sequence } \\
(N=102)\end{array}$ & $\begin{array}{l}214 \\
(79)\end{array}$ & $\begin{array}{c}237 \\
(100)\end{array}$ & $\begin{array}{c}5.0 \\
(1.8)\end{array}$ & $\begin{array}{c}2.1 \\
(1.5)\end{array}$ \\
\hline
\end{tabular}

first fixation of RS, second fixation of PS. With the exception of the two fixations at the word end, all these positions were significantly different from each other (see Kliegl, 1981b, for details). Thus, during a PS the fixations are closer to the beginning of a word than during a RS. Also, the position of the fixation in the OFC is between the first and last fixation of the TFC.

The distinction between a progressive and a regressive sequence in the twofixation case revealed differences that are plausible in the context of an asymmetry of processing in the visual field. Recent research shows this field to extend up to 15 characters to the right (O'Regan, 1979; Rayner \& Bertera, 1979; Rayner, Inhoff, Morrison, Slowiaczek, \& Bertera, 1981; Rayner, McConkie, \& Ehrlich, 1978) and about 4 letters to the left (Rayner, McConkie, \& Zola, 1980; Rayner, Well, \& Pollatsek, 1980). The positions of the fixations and their variation with word length indicate that with a fixation at the beginning of a word, most letters will be processed from this location. The few remaining peripheral letters cause the second fixation to fall close to the end of the word and allow for a shorter fixation at this location.

The data summarized in Table 19.2 were obtained from words ranging from five to nine letters in length. It was shown earlier that word length had no effect on fixation time for words receiving a single fixation. The same result was obtained for the two-fixation condition. However, the relevance of word length for eye-guidance factors could be demonstrated if there were a dependence between fixation position and word length. Fixations too close to either end of a word may necessitate a second fixation, thereby nearly doubling the total fixation time. Several polynomial analyses were executed for the letter positions of the various fixations. Significant linear trends were found for the position of the last fixation in a progressive sequence, for both the fixations in a regressive sequence, and for the one-fixation condition. With the exception of the first fixation in a progressive sequence, fixation positions tended to shift toward the right as word length increased. 
The two fixation conditions are certainly in agreement with what one would expect under peripheral acuity limitations. However, there is the possibility that the tendency to make a second fixation is related to psycholinguistic processing demands of the word. To test this hypothesis, the average word frequency was calculated as a function of number of fixations for each of the three submatrices indicated in Table 19.1. In all three tests word frequency was lower when two fixations occured, but the difference was significant only for the words 10 and 11 letters long. Thus, we again find some evidence, for the effect of word frequency on eye movements.

\section{Influences across Words}

So far we presented the problem of perceptual acuity limitations and language processing as a problem to identify what proportion of fixation duration variance is allocated to either of them. Our data provide relatively weak evidence for the relevance of language processes. Summing fixations on a word into a gaze measure and interpreting this time merely as a reflection of psycholinguistic processes as Just and Carpenter (1980) propose, seems not warranted in the light of our analysis on convenient viewing. If a second fixation occurs for perceptual reasons, a large proportion of the second fixation reflects the time it takes to generate a second saccade and determine its ballistic properties. Nevertheless, there might be a subtle interaction between achieving a convenient position on a word and the demands for psycholinguistic processing resources preceding this word. That is, perceptual acuity limitations might arise due to psycholinguistic processing demands. If a portion of the text requires intensive psycholinguistic processing, the quality of peripheral preprocessing might suffer and lead to saccades that are less than optimal in positioning the next fixation. We are presently designing experiments that will test the influence of cognitive processing demands on the accuracy of the saccade generating system.

An alternative explanation for the occurrence of two fixations due to inconvenient viewing position is the saccade generating system itself. We will test two possible hypotheses related to this explanation. First, inconvenient viewing positions might arise due to serial dependencies in the saccade and fixation duration series. Second, there might be a lack of resilience to make very long or very short saccades. Under situations where this would be required to obtain the convenient position, an undershoot or overshoot might result.

\section{A. Serial Dependencies}

As eye movements and fixations form a time series, an obvious question to ask from these data is whether subsequent fixation durations and saccade sizes show an autocorrelational structure. Further, one wonders whether there are any crosscorrelations between these two parameters. If the length of a saccade were depen- 
dent on the length of preceding saccades, or on the duration of previous fixations, this would lead to inaccurate placements of fixations on a word. For example, if saccade $\mathrm{N}$ was long, saccade $\mathrm{N}+\mathrm{I}$ might be short. These dependencies could theoretically exist independent of word and text characteristics and override the preprocessing abilities of the saccade calculating system.

There have been several studies looking for a correlation between and within the eye movement parameters. Rayner and McConkie (1976) came to the conclusion that saccade size and fixation duration are two factors that must be accounted for separately. Their basis for this is the lack of correlation between successive fixation durations, between successive saccade sizes, and between the size of a saccade and the preceding and following fixation time, respectively. Poor correlations among similar variables have been reported by Andriessen and de Voogd (1973). Further, Just and Carpenter (1980) did not find significant autocorrelations for the residuals of their data.

It is worth pointing out that all the studies reviewed only calculated the lag-one autocorrelation. Thus, it is not clear whether there are some higher order relations. To provide both a replication and an extension of the previous reports the complete autocorrelation functions were calculated. The results indicated that neither for fixation time, that is, the sum of the fixations on a word, nor the first fixation durations, nor for the saccade sizes out of a word were there significant correlations at any lag. Taking the sequence of fixations as the series-instead of the sequence of words-yielded similar results. Thus, at a global level, these results are in agreement with previous research and yield similar results for higher order correlations. They also do not support the hypothesis that serial dependencies might be a reason for inconvenient viewing positions.

\section{B. Lack of Saccadic Resilience}

The analysis of the one-fixation words showed that there was a strong tendency to fixate close to the center of a word. In regression terminology, word length was a good predictor of the fixation position on the word. Since saccades are ballistic movements, this is strong support for preprocessing of peripheral information. However, word length of the fixated word does not explain all of the fixation position variance. The position of a fixation might also be determined by the position on and the length of the previous word. For example, given a fixation at the end of a word, one could expect the next fixation to be also close to the end of the word. Or, given a long word and a middle fixation position, one might expect the following fixation position to be closer to the beginning of the word. Basically, it is assumed that saccade size lacks the required resilience to counteract any misplacements that might arise because of the partly stochastic nature of the saccade generating system. Consequently, nonoptimal positions might be obtained because of characteristics of word and position during the previous fixation. To test for the influence of the previous word length and previous fixation position, we calculated multiple correlations between the fixation position and length of the 
TABLE 19.3

Multiple Correlation of Fixation Position with Length of Previous Word and Position of Previous Fixation

\begin{tabular}{lcccccc}
\hline Subject & 1 & 2 & 3 & 4 & 5 & 6 \\
\hline$R^{a}$ & .20 & .35 & .10 & .32 & .20 & .24 \\
$\mathrm{~N}^{b}$ & 125 & 231 & 256 & 172 & 127 & 193 \\
\hline
\end{tabular}

${ }^{a} R$ is the multiple correlation between position of fixation and length of and position of fixation on previous word (length of present word partialed out).

${ }^{b} N$ is the number of observations.

previous word, and the fixation position on the current word. We first partialed out the length of the current word. The only data included were word pairs that were next to each other in the text for which the eye progressed from the first to second word. The results are given in Table 19.3. The incremental $R$ 's are significant for all but the third subject. The correlations confirm our expectations that the current fixation position is dependent on both the length of the previously fixated word and the fixation position of that word. The results suggest that the saccade generating system occasionally cannot generate the very short or very long saccades that are sometimes required to achieve an optimal viewing position. Fixations following long words tend to be at the beginning of the word; fixations following short words tend to be toward the end.

\section{Conclusion}

This chapter presented some thought and data about the possibilities of unconfounding perceptual and language processes. In the first set of analyses on singlefixation words, length was not related to fixation duration for words ranging from 5 to 11 letters and one to four syllables. These results are inconsistent with the syllable-unit processing proposed by Just and Carpenter (1980) and Gough's (1972) serial letter-scanning model. The lack of a word-length effect on single fixation durations is consistent with word-unit and parallel letter processing models (cf. Cattell, 1885; McClelland \& Rumelhart, 1981). It also is in accord with research on semantic classification and lexical decision. For example, Terry, Samuels, and LaBerge (1976) found that in a semantic classification task, word length had no effect on decision time. However, the lack of word length effects has only been shown for reasonably well-known words in a semantic context. Infrequent and novel words, beginning readers, and poor readers might yield different results.

In the second set of analyses, previous experimental results by Rayner (1979a) and O'Regan $(1980,1981)$ on the "preferred" and "convenient" position of fixations were replicated. The number of fixations on a word were interpreted to be mostly due to perceptual factors. For long words, however, the frequency of words 
that received two fixations was lower than for words of the same length fixated once. This corresponds to a result from the analysis of single-fixation words wherein fixation duration correlated at .11 with word frequency. Thus, fixation time appeared to be influenced by perceptual factors related to acuity limits in eye guidance, but there was also evidence for the relevance of psycholinguistic variables.

Kliegl et al. (1982) demonstrated that multiple regression approaches as the one advanced by Just and Carpenter (1980) are critically dependent on multiple fixations of words. The analyses of two-fixation cases from the perspective of inconvenient viewing called into question the justification of summing fixations on a word into a measure of gaze. It seems necessary to explore the causes of inconvenient viewing that then lead to the potential doubling of fixations. Although O'Regan's (1980, 1981) experimental induction, that is, shifting a word during a saccade, was ideal for demonstrating the relevances of an inconvenient position on a word, the "origin" of inconvenient viewing positions under conditions of normal reading is still an open question. The final part of this chapter tested two possible causes that would result as consequences of properties assumed for the saccade generating system. The first hypothesis, serial dependencies of subsequent saccades, was not confirmed by the data. However, there was support for the second hypothesis: The position of the fixation in a word can be predicted to some degree by the length of the previous word and the position of the fixation on this word. The data were consistent with an assumption that saccades do not tend to be very short or very long, even if this were required to achieve the optimal viewing position within a word. Thus, the doubling of fixations may have its cause in an inconvenient positioning of a fixation on a previous word. In a sense, only an "aggregation of inconvenience" might lead to doubling up.

There is, of course, the possibility that psycholinguistic processing demands will cause the saccade generating system to operate less accurately. This hypothesis predicts that the doubling up of fixations would occur in regions of the text that generate greater cognitive demands. The more we can determine the influences of previous conditions on present ones, the closer we will come to an understanding of the dynamics of the reading process.

In conclusion, the results argue for the consideration of both perceptual and psycholinguistic processes in the evaluation of eye fixation data. Further, the more challenging tasks will lie in exploring the interaction of the saccade generating system with the cognitive processing demands during reading. This will considerably enhance the value of these data for developing a flexible process model of reading that accounts for variations in individuals, materials, and task demands.

\section{References}

Andriessen, J. J., \& de Voogd, A. H. (1973). Analysis of eve-movement patterns in silent reading. IPO Annual Progress Report, 8, 29-53.

Carnus, H. (1948). The plague (transl. by S. Gilbert). New York: Random House. (Originally published 1947.) 
Cattell, J. McK. (1885). Ueber die Zeit der Erkennung und Bennenung von Schriftzeichen, Bildern, und Farben. Philosophische Studien, 2, 635-650.

Davidson, B. J. (1981). A rotating buffer system for on-line collection of eye monitor data. Behavioral Research Methods and Instrumentation, 13, 112-114.

Gough, P. B. (1972). One second of reading. In J. F. Kavanaugh \& I. G. Mattingly (Eds.), Language by ear and by eye. Cambridge, Massachusetts: MIT Press.

Just, M. A., \& Carpenter, P. A. (1980). A theory of reading: From eye fixations to comprehension. Psychological Review, 87, 329-354.

Kliegl, R. (1981). Automated and interactive analysis of eye fixation data in reading. Behavioral Research Methods and Instrumentation, 13, 115-120. (a)

Kliegl, R. (1981). Eye-movements in reading: An attempt to separate perceptual and psycholinguistic factors. Unpublished master's thesis, University of Colorado. (b)

Kliegl, R., \& Olson, R. K. (1981). Reduction and calibration of eye monitor data. Behavioral Research Methods and Instrumentation, 13, 107-111.'

Kliegl, R., Olson, R. K., \& Davidson, B. J. (1982). Regression analysis as a tool for studying reading processes: Comment on Just and Carpenter's eye fixation theory. Menory \& Cognition, 10,287-95.

McClelland, J. L. \& Rumelhart, D. E. (1981). An interactive activation model of context effects in letter perception: Part 1. An account of basic findings. Psychological Review, 88, 375-407.

McConkie, G. W. (1979). On the role and control of eye movements in reading. In P. A. Kolers, M. E. Wrolstad, \& H. Bouma (Eds.), Processing of visible language. New York: Plenum Press.

O'Regan, J. K. (1979). Saccade size control in reading: Evidence for the linguistic control hypothesis. Perception \& Psychophysics, 17, 578--586.

O'Regan, J. K. (1980). The control of saccade size and fixation duration in reading: The limits of linguistic control. Perception \& Psychophysics, 28, 112-117.

O'Regan, J. K. (198I). The "convenient viewing position" hypothesis. In D. F. Fisher, R. A. Monty, \& J. W. Senders (Eds.), Eye movements: Cognition and visual perception. Hillsdale, New Jersey: Erlbaum.

Pynte, J. (1974). Readiness for pronunciation during the reading process. Perception \& Psychophysics, $16,487-504$.

Rayner, K. (1979). Eye guidance in reading: Fixation-locations within words. Perception, 8, 21-30. (a)

Rayner, K. (1979). Eye movements in reading: Eye guidance and integration. In P. A. Kolers, M. E. Wrolstad, \& H. Bouma (Eds.), Processing of visible language. New York: Plenum Press. (b)

Rayner, K., \& Bertera, J. H. (1979). Reading without a fovea. Science, 206, 468-469.

Rayner, K., Inhoff, A. W., Morrison, R. E., Slowiaczek, M. L., \& Bertera, J. H. (1981). Masking of foveal and parafoveal vision during eye fixations in reading. Journal of Experimental Psychology: Human Perception \& Performance, 7, 167-179.

Rayner, K., \& McConkie, G. W. (1976). What guides a reader's eye movements? Vision Research, 16, $829-837$.

Ravner, K., McConkie, G. W., \& Ehrlich, S. (1978). Eye movements and integrating information across fixations. Joumal of Experimental Psychology: Human Perception \& Performance, 4, $529-544$.

Rayner, K., McConkie, G. W., \& Zola, D. (1980). Integrating information across eve movements. Cognitive Psychology, 12, 206-226.

Rayner, K., Well, A. D., \& Pollatsek, A. (1980). Asymmetry of the effective visual field in reading. Perception \& Psychophysics, 27, 537-544.

Terry, P., Samuels, S. J., \& LaBerge, D. (1976). The effect of letter degradation and letter spacing on word recognition. Joumal of Verbal Learning and Verbal Behavior, 15, 577-585.

Tinker, M. A. (1958). Recent studies of eye movements in reading. Psychological Bulletin, 55, $215-231$. 